\title{
Target Location Algorithm by Acoustic Energy-Ratio and Experimental Research
}

\author{
Tang Yonggang, Luo Hu, Shen Jun, Fu Xinhua \\ Air Force Airborne Academy, Guilin, China \\ e-mail:tygor@163.com
}

\begin{abstract}
Keywords: acoustic;energy-ratio;location;distributed; wireless sensor network
Abstract. A target location algorithm for distributed acoustic sensors was presented. Acoustic energy-ratio between sensors was used to solved the position of sound source iteratively, without priori information of position and rigorous clock synchronization. Experiments showed that target could be accurately located. The algorithm can run smoothly on low-cost wireless sensor networks nodes for its simplicity and efficiency.
\end{abstract}

\section{Introduction}

Wireless sensor networks (WSN) is a rapidly developed technology which integrates sensor technology with wireless communication, embedded computing and distributed information processing. Usually WSN sensor nodes are low-cost and small in size, with short-distance communication capability to each other. Mass sensor nodes can be widely distributed in large area and self-organized into a network. Multiple sensor nodes can cooperate to surveillance, percept, sample various data of environment and targets. By processing the collected data, detailed and accurate information can be acquired and delivered to end users. WSN technology is now widely used in various areas such as defense, safety surveillance, environment monitoring, traffic control as well as disaster prevention and relief [1].

Target location is an important research field of WSN. Compared with radio signal, acoustic signal has lower frequency, slower spreading speed as well as longer propagation distance and stronger diffraction ability. The cost and complexity of a sound sensor is much lower than other sensors so acoustic localization and identification is popular in applications of wireless sensor networks [2].

Substantially sound is a kind of mechanical oscillation. It can broadcast through liquid, solid and gas from its source in all directions. The intension and spectrum of sound wave is affected by the position of sound source, movement, media on the route and the structure of sound source itself. Using acoustic sensors we can receive sound wave and draw the information of its source. To locate a sound source there is no need to send out signals so this method can be applied all passively [3].

In a general way multiple sound sensors are essential for target location. Location approaches can be divided into two groups. The first is interaural time difference (ITD) or interaural phase difference (IPD) based technique. The time or phase of arrival differs from nodes in different distance. By processing data from multiple nodes we can determine the location of sound source utilizing the relative shifts in the signals received by microphones. For example, the methods of time-delay estimation (TDE) [4-6], beam forming [7-9], hemisphere sampling [10], and accumulated correlation $[11,12]$ were different ways of this type [13]. However, by this kind of approaches rigorous time synchronization among all nodes was essential. Spatially separated WSN nodes with independent clocks are difficult to assure positioning accuracy by synchronizing the received data.

The second group refers to energy based approaches. The signals received by microphones differ in their intensity level, with a microphone closer to the sound source receiving a higher intensity signal than a farther microphone. By measuring the intensity level of received signals using multiple nodes we can find out the position of sound source. This kind of techniques does not require accurate time synchronization, so we can simplify pre-processing steps and use the average energy of the signals over a relative large window to depress noises. Birchfield gave a location method based on 
interaural level difference(ILD) [14]. ILD method computed the sound source location by combining likelihood functions, one for each microphone pair. Nevertheless, this developed methods mentioned above, had some limitations:

A number of candidate locations is needed. Few candidate locations lead to poor accuracy while too many candidates will bring large burden of computation.

Limited number of candidate locations can only cover limited area, so this method have to be used in a definite bound of area, for example, a meeting room.

The signal noise is required to follow normal distribution and its first as well as second moment should be known beforehand. In fact received signals are always polluted by colored noises so that it is hard to meet the requirement mentioned above.

To suffice the need for target location using large number of randomly distributed sound sensor nodes, an algorithm based on iterative least square solution is presented. By this method there is no need of selecting candidate locations and prior information about the received signal as well as sound source. It is demonstrated in experiments that this algorithm has low calculation burden and converges fast. The accuracy is good enough to fulfill the requirement of target location mission for WSN.

\section{Location Algorithm}

In most situations the original intensity level of sound source is unknown. It is hard to estimate the position of sound source by directly measuring its energy. However, If the signal is sent from one source, the energy-ratio between different nodes will only rest with relative distance between the nodes and sound source, so we can use energy-ratio between every two nodes as the measurement.

\section{Reference Frame}

Selecting any fixed point on the plane of nodes as the origin, a Cartesian plane XY-coordinate system is established as shown in Fig. 1. Assume there be $\mathrm{n}$ nodes in this network and their position be known as $\left(x_{1}, y_{1}\right),\left(x_{2}, y_{2}\right), \ldots \ldots,\left(x_{n}, y_{n}\right)$ respectively. Let $(x, y)$ be the coordinates of target (sound source).

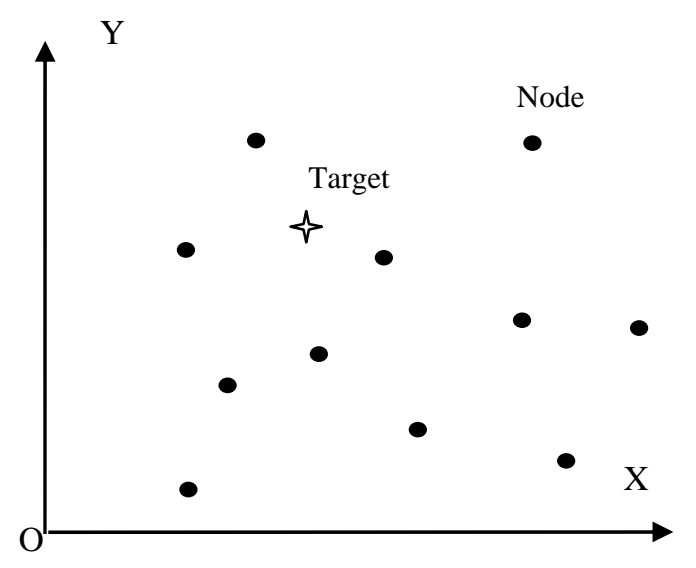

Figure 1. Reference Frame for Location

\section{Energy-ratio measurement equation}

For energy measurement time delay is negligible, the signal received by the ith nodes can be modeled as [14]

$$
r_{i}(t)=s(t) / d_{i}+\xi_{i}(t)
$$

where $s(t)$ is the signal sent by sound source, $d_{i}$ is the distance from the source to the ith node, $\xi_{i}(t)$ is the noise of measurement. Suppose that the sound source is audible during a time interval $[0, \mathrm{~W}]$, energy received by the i-th node can be obtained by integrating the square of the signal over this time interval: 


$$
E_{i}=\int_{0}^{w} r_{i}^{2}(t) d t
$$

From (1) and (2) we get

$$
E_{i}=\frac{1}{d_{i}^{2}} \int_{0}^{w}\left[s^{2}(t)\right] d t+\int_{0}^{w} \xi_{i}^{2}(t) d t
$$

Given two nodes $\mathrm{i}$ and $\mathrm{j}$, we have

$$
E_{i} d_{i}^{2}=E_{j} d_{j}^{2}+\eta_{i j}
$$

where $\eta_{i j}=\int_{0}^{w}\left[\xi_{i}^{2}(t)-\xi_{j}^{2}(t)\right] d t$. Substituting

$$
\begin{gathered}
d_{i}{ }^{2}=\left(x-x_{i}\right)^{2}+\left(y-y_{i}\right)^{2} \\
d_{j}{ }^{2}=\left(x-x_{j}\right)^{2}+\left(y-y_{j}\right)^{2}
\end{gathered}
$$

into (4) , after some algebraic manipulation we have

$$
\left(x-\frac{c_{i j}^{(x)}}{c_{i j}^{(e)}}\right)^{2}+\left(y-\frac{c_{i j}^{(y)}}{c_{i j}^{(e)}}\right)^{2}=\frac{E_{i} E_{j} d_{i j}{ }^{2}}{\left(c_{i j}^{(e)}\right)^{2}}+\eta_{i j}{ }^{\prime},
$$

where

$$
\begin{gathered}
c_{i j}^{e}=E_{i}-E_{j} \\
c_{i j}^{(x)}=E_{i} x_{i}-E_{j} x_{j} \\
c_{i j}^{(y)}=E_{i} y_{i}-E_{j} y_{j} \\
d_{i j}=\left(x_{i}-x_{j}\right)^{2}+\left(y_{i}-y_{j}\right)^{2}
\end{gathered}
$$

From the equation above we can get an equation set:

$$
\left\{\begin{array}{c}
\left(x-\frac{c_{12}^{(x)}}{c_{12}^{(e)}}\right)^{2}+\left(y-\frac{c_{12}^{(y)}}{c_{12}^{(e)}}\right)^{2}=\frac{E_{1} E_{2} d_{12}{ }^{2}}{\left(c_{12}^{(e)}\right)^{2}}+\eta_{12}{ }^{\prime} \\
\left(x-\frac{c_{13}^{(x)}}{c_{13}^{(e)}}\right)^{2}+\left(y-\frac{c_{13}^{(y)}}{c_{13}^{(e)}}\right)^{2}=\frac{E_{1} E_{3} d_{13}{ }^{2}}{\left(c_{13}^{(e)}\right)^{2}}+\eta_{13}{ }^{\prime} \\
\ldots \\
\left(x-\frac{c_{1 n}^{(x)}}{c_{1 n}^{(e)}}\right)^{2}+\left(y-\frac{c_{1 n}^{(y)}}{c_{1 n}^{(e)}}\right)^{2}=\frac{E_{1} E_{n} d_{1 n}{ }^{2}}{\left(c_{1 n}^{(e)}\right)^{2}}+\eta_{1 n}{ }^{\prime}
\end{array}\right.
$$

\section{Sulution of source position}

For any pair of nodes, if the energy-ratio $\Delta E_{i j}=E_{i} / E_{j} \neq 1$, According to (5) the sound source is constrained to lie on a circle centered at $\left(c_{i j}^{(x)} / c_{i j}^{(e)}, c_{i j}^{(y)} / c_{i j}^{(y)}\right)$ with a radius of $\sqrt{E_{1} E_{2}} d_{i j} / c_{i j}^{(e)}$. If the energy-ratio $\Delta E_{i j}=1$, then this circle will converge to a midline, as shown in Fig. 2. 


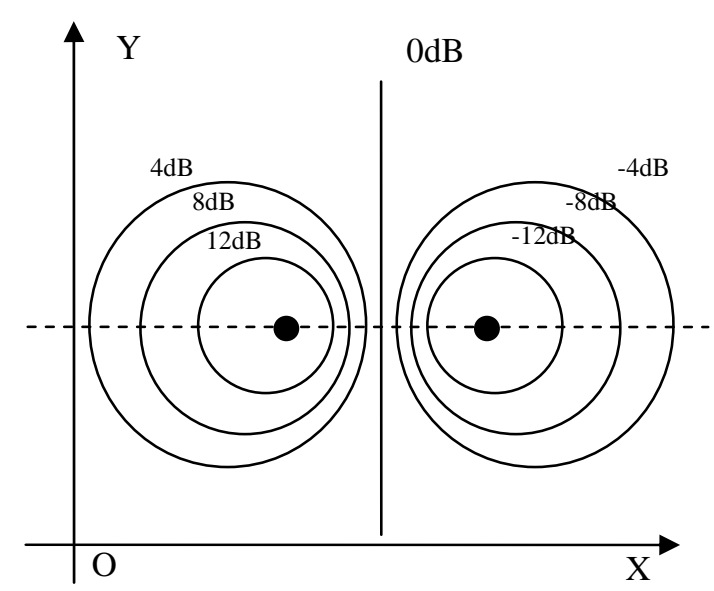

Figure 2. Isocontours of sound source position

As shown in Fig. 3, in a practical sensor networks system, if there are 4 sensors which are not distributed on a line have received acoustic signals from a same target, then there will be at least 3 different isocontours which have only one point of intersection. This intersection is corresponding to the position of sound source, namely, the equation set in (6) have exclusive solution. If there are more sensors can receive this signal, redundant measurements are available and help in reducing affection

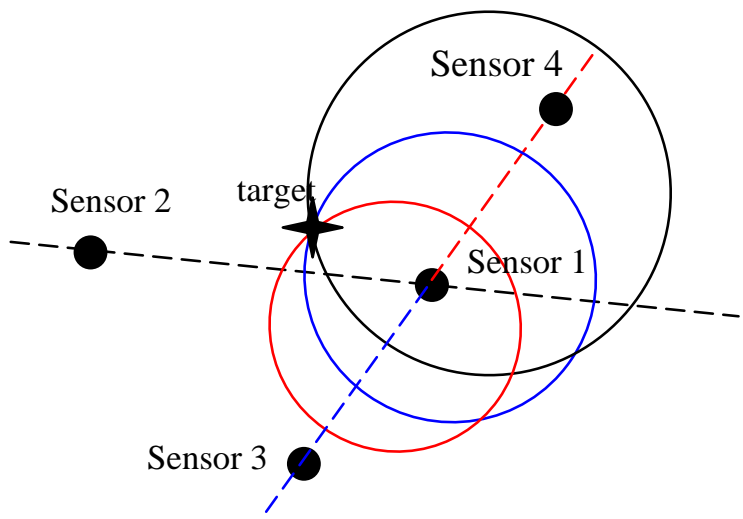

by noises.

Figure 3. Geometrical explaination for existance and exclusivity of solution

Whereas computation resources of WSN sensor nodes are scarce, simple approaches on solving equation set (6) have to be found. Ignoring noises, the equation for a pair of sensors $\mathrm{i}$ and $\mathrm{j}$ can be written as

$$
\frac{E_{i}}{E_{j}}=\frac{d_{j}^{2}}{d_{i}^{2}}=\frac{\left(x-x_{j}\right)^{2}+\left(y-y_{j}\right)^{2}}{\left(x-x_{i}\right)^{2}+\left(y-y_{i}\right)^{2}}
$$

Using log function on both sides we have

$$
\begin{aligned}
\ln \frac{E_{i}}{E_{j}} & =\ln \left[\left(x-x_{j}\right)^{2}+\left(y-y_{j}\right)^{2}\right] \\
& -\ln \left[\left(x-x_{i}\right)^{2}+\left(y-y_{i}\right)^{2}\right]
\end{aligned}
$$

Assuming initial value of target position be $\left(x_{0}, y_{0}\right)$, and the position error be $(\delta x, \delta y)$, we have $x=x_{0}+\delta x, y=y_{0}+\delta y$. Expanding (8) into tylor series: 


$$
\begin{aligned}
\ln \frac{E_{1}}{E_{2}} & =\ln \left[\left(x_{0}-x_{2}\right)^{2}+\left(y_{0}-y_{2}\right)^{2}\right] \\
& -\ln \left[\left(x_{0}-x_{1}\right)^{2}+\left(y_{0}-y_{1}\right)^{2}\right] \\
& +\frac{2\left(x_{0}-x_{2}\right)}{\left(x_{0}-x_{2}\right)^{2}+\left(y_{0}-y_{2}\right)^{2}} \delta x \\
& +\frac{2\left(y_{0}-y_{2}\right)}{\left(x_{0}-x_{2}\right)^{2}+\left(y_{0}-y_{2}\right)^{2}} \delta y \\
& -\frac{2\left(x_{0}-x_{1}\right)}{\left(x_{0}-x_{1}\right)^{2}+\left(y_{0}-y_{1}\right)^{2}} \delta x \\
& -\frac{2\left(y_{0}-y_{1}\right)}{\left(x_{0}-x_{1}\right)^{2}+\left(y_{0}-y_{1}\right)^{2}} \delta y
\end{aligned}
$$

After some algebraic manipulation we have

$$
\begin{aligned}
\frac{1}{2}\left(\ln \frac{E_{1}}{E_{2}}-\ln \frac{d_{2}^{2}}{d_{1}^{2}}\right) & =\left[\frac{\left(x_{0}-x_{2}\right)}{d_{2}^{2}}-\frac{\left(x_{0}-x_{1}\right)}{d_{1}^{2}}\right] \delta x \\
& +\left[\frac{\left(y_{0}-y_{2}\right)}{d_{2}^{2}}-\frac{\left(y_{0}-y_{1}\right)}{d_{1}^{2}}\right] \delta y
\end{aligned}
$$

where

$$
\begin{aligned}
& d_{1}^{2}=\left(x_{0}-x_{1}\right)^{2}+\left(y_{0}-y_{1}\right)^{2}, \\
& d_{2}^{2}=\left(x_{0}-x_{2}\right)^{2}+\left(y_{0}-y_{2}\right)^{2} .
\end{aligned}
$$

If there are $\mathrm{n}$ sensors, we can get a $\mathrm{n}-1$ dimension equation set. Let

$$
\begin{gathered}
X=\left[\begin{array}{ll}
\delta x & \delta y
\end{array}\right]^{T} \\
Z=\left[\begin{array}{cc}
\ln \frac{E_{1}}{E_{2}}-\ln \frac{d_{2}^{2}}{d_{1}^{2}} \\
\ln \frac{E_{1}}{E_{3}}-\ln \frac{d_{3}^{2}}{d_{1}^{2}} \\
\cdots \\
\ln \frac{E_{1}}{E_{n}}-\ln \frac{d_{n}^{2}}{d_{1}^{2}}
\end{array}\right] \\
V=\left[\begin{array}{llll}
v_{12} & v_{13} & \cdots & v_{1 n}
\end{array}\right]^{T}
\end{gathered}
$$

we have

$$
Z=H X+V
$$

The least square estimation of position errors should be

$$
\hat{X}=\left(H^{T} H\right)^{-1} H^{T} Z
$$

The estimated position of acoustic source is

$$
\begin{aligned}
& \hat{x}=x_{0}+\delta \hat{x} \\
& \hat{y}=y_{0}+\delta \hat{y}
\end{aligned}
$$

To get a satisfying solution, (11) can be solved iteratively until $\delta \hat{x}$ and $\delta \hat{y}$ lower than threshold value. 


\section{Target tracing}

Suppose computational time step length is $T$, current time is $t_{k}$, the position of target at previous two time steps are $\hat{\bar{p}}\left(t_{k-1}\right)$ and $\hat{\bar{p}}\left(t_{k-2}\right)$ respectively. The velocity of target can be estimated as

$$
\begin{gathered}
\hat{\vec{v}}\left(t_{k}\right)=\left[\hat{\bar{p}}\left(t_{k-1}\right)-\hat{\bar{p}}\left(t_{k-2}\right)\right] / T \quad \text { (13) } \\
\quad \text { Current position of target at time can be forecasted as } \\
\tilde{\tilde{p}}\left(t_{k}\right)=\hat{\vec{v}}\left(t_{k}\right) \cdot T
\end{gathered}
$$

Using $\tilde{\bar{p}}\left(t_{k}\right)$ as initial value, namely

$$
\begin{aligned}
& x_{0}\left(t_{k}\right)=2 \hat{x}\left(t_{k-1}\right)-\hat{x}\left(t_{k-2}\right) \\
& y_{0}\left(t_{k}\right)=2 \hat{y}\left(t_{k-1}\right)-\hat{y}\left(t_{k-2}\right)
\end{aligned}
$$

By iteratively solving (11) we can get current position of target.

\section{Experiments And Results}

Field tests were carried out to examine the algorithm. 4 acoustic sensor nodes and 1 sink node which self-organized into a sensor networks were used in tests. The distribution of nodes are shown in Fig. 4.

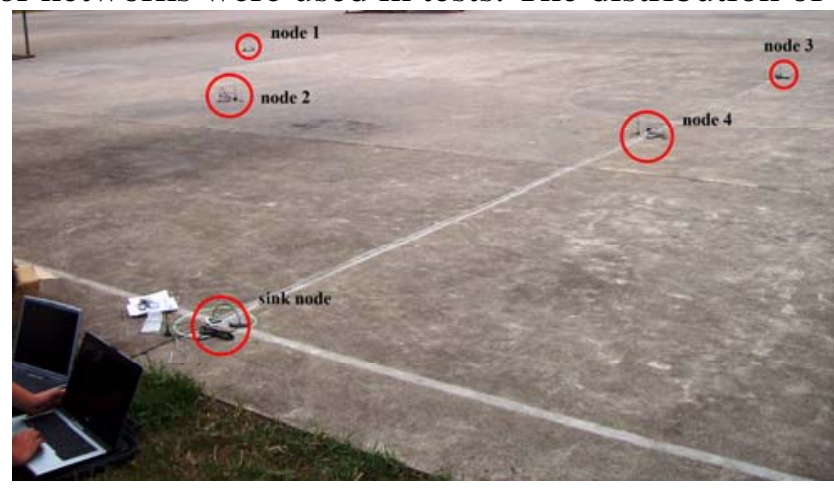

Figure 3. Distribution of nodes in tests

Each node was equipped with a ARM7TDMI CPU(AT91SAM7S256), a wireless communication chip CC1100, and a microphone whose signal was amlified by OPA2349 and then sampled on 8KHz frequency. A motocycle with a satellite enhanced Novatel OEM4 GPS receiver was used as target. Position outputs with an accuracy better than $1 \mathrm{~m}(\mathrm{CEP})$ from the GPS receiver were used as reference.

The initial position of target was defined as original point. The target started from point $(0,0)$, moving northeast, then turning to northwest, and stopped at point $(26.5,288.5)$. The trajectory and computation results are shown in Fig. 5., where 4 triangles are position of 4 nodes, dashed line is referenced GPS target trajectory, round points are results of position estimation.

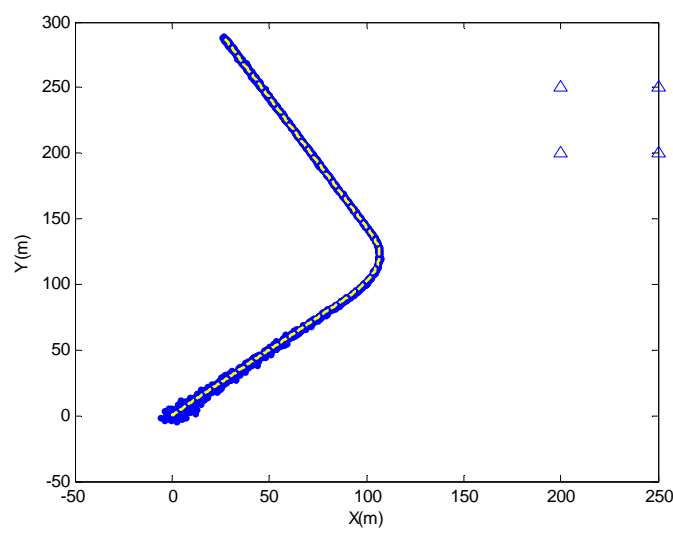

Figure 4. Target trajectory and locating results 
In Fig. 6 an enlarged section of the trajectory is shown. It is obvious that the estimated points is distributed close to the referenced trajectory. The position errors are shown in Fig. 7. It can be seen that the largest errors are about $5 \mathrm{~m}$ and their values raise with the average distance from the target to nodes.

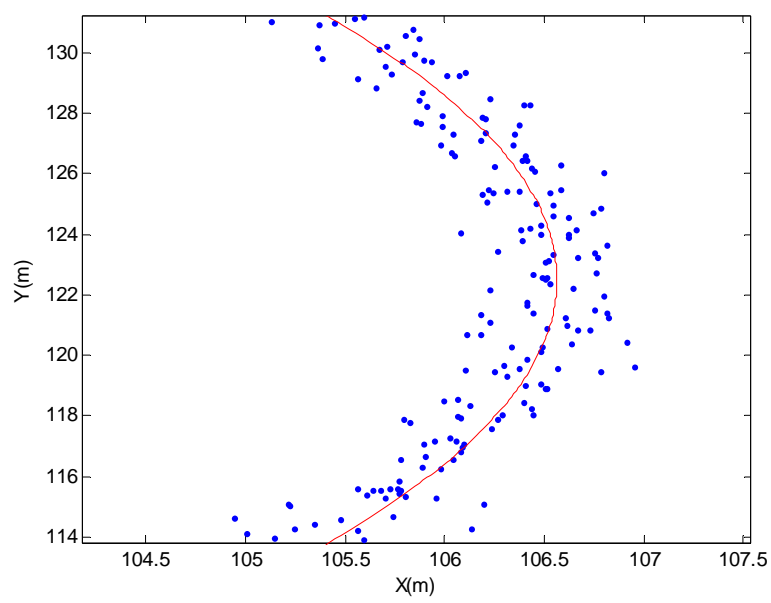

Figure 5. Comparision between referenced trajectory and estimating resultes
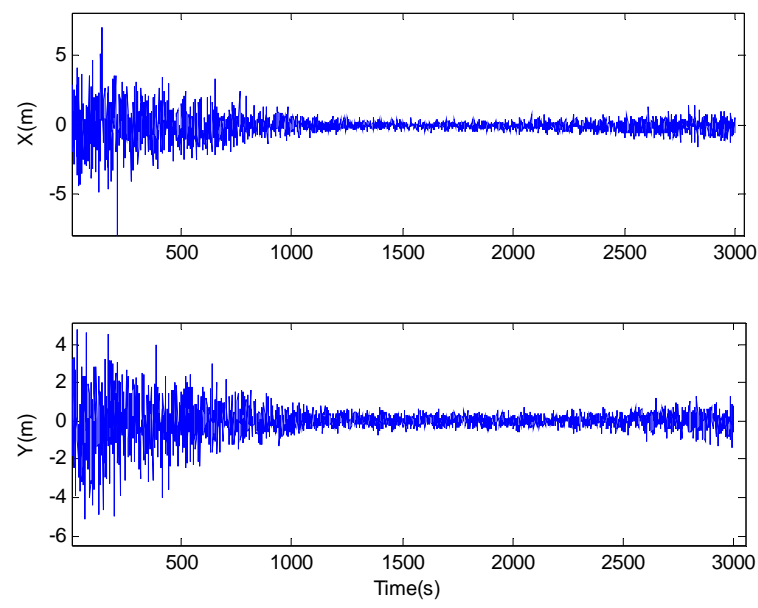

Figure 6. Errors between referenced trajectory and estimated positon

\section{Conclusion}

The algorithm presented in this article is quite simple. It has no requirement on priori information about target position nor rigorous clock synchronization among nodes. Low computation loads makes it run smoothly on low-cost nodes with few memory and low computation capability. In experiments this algorithm was applied successfully on a simple self-organized wireless sensor networks. It was shown that the estimated position was accurate enough to trace the movement of target. It is expected to apply this method on wireless sensor networks which have large amount of low-cost nodes distributed in wide areas. 


\section{References}

[1] T. Arampatzis, J. Lygero, and S. Manesis, A Survey of Applications of Wireless Sensors and Wireless Sensor Networks, Mediterranean Conference on Control and Automation, Limassol, Cyprus, 2005.

[2] W. Hongkai, F. Liqing, X. Chao, and L. Xianfeng, On Issues of Acoustic Passive Location Algorithm, International Conference on Instrumentation, Measurement, Computer, Communication and Control, 2011.

[3] L. Tong and W. Lu, Technique of Acoustic Location and Its Military Application, International Conference on Information Acquisition, 2004.

[4] M. S. Brandstein and H. F. Silverman, Practical Methodology for Speech Source Localization with Microphone Arrays, Computer Speech and Language, 11(2): 91-126, 1997.

[5] P. Svaizer, M. Matassoni, and M. Omologo, Acoustic Source Location in a Three Dimensional Space using Crosspower Spectrum Phase, Proceedings of the IEEE International Conference on Acoustics, Speech, and Signal Processing, 1997, pp. 231-234.

[6] J. Vermaak and A. Blake, Nonlinear Filtering for Speaker Tracking in Noisy and Reverberant Environments, ICASSP, 2001.

[7] R. Duraiswami, D. Zotkin, and L. Davis, Active Speech Source Localization by a Dual Coarseto-fine Search, ICASSP, 2001.

[8] D. B. Ward and R. C. Williamson, Particle Filter Beamforming for Acoustic Source Localization in a Reverberant Environment, ICASSP, 2002.

[9] H. Tian, X. Deng-Hong, L. Xian-Dong, and S. Ying-Chun, Research of Acoustic Emission Source Location Based on Near-field Beamforming, Journal of Vibration Engineering, 25(2): 199-205, 2012.

[10] S. T. Birchfield and D. K. Gillmor, Acoustic Source Direction by Hemisphere Sampling, ICASSP, 2001.

[11] S. T. Birchfield and D. K. Gillmor, Fast Bayesian Acoustic Localization, ICASSP, 2002.

[12] S. T. Birchfield, A Unifying Framework for Acoustic Localization, Proceedings of the 12th European Signal Processing Conference, 2004.

[13] S. T. Birchfield and R. Gangishetty, ACOUSTIC LOCALIZATION BY INTERAURAL LEVEL DIFFERENCE, ICASSP 2005, 2005.

[14] W. Cui, Z. Cao, and J. Wei, DUAL-MICROPHONE SOURCE LOCATION METHOD IN 2D SPACE, ICASSP 2006, 2006. 